Enfermagem Brasil 2018;17(3):175-81

\title{
ARTIGO ORIGINAL \\ Perfil epidemiológico da sífilis congênita em Salvador: 2007-2016
}

Tatiane Sena*, Tatiane dos Santos Pessoa*, Edla Queiroz Brito**, Edvânia Matos de Oliveira*, Flavia Pimentel Miranda, M.Sc..**

*Enfermeira, Pós-graduanda em pediatria e neonatologia pela Escola Bahiana de Medicina e Saúde Pública, Salvador/BA, ${ }^{* *}$ Enfermeira, graduada pela UNIFACS-Universidade Salvador, Salvador/BA, ${ }^{* \star}$ Enfermeira, Professora Assistente I da UNIFACS - Universidade Salvador

Recebido em 10 de outubro de 2017; aceito em 21 de março de 2018.

Endereço para correspondência: Flavia Pimentel Miranda, Rua Emílio Odebrecht, 79/402, Ed. Praia Rasa Pituba, 41830-300 Salvador BA, E-mail: flaviabrim@hotmail.com, Tatiane Sena: tsena8745@gmail.com; Tatiane dos Santos Pessoa: tatypessoa01@gmail.com; Edla Queiroz Brito: edla.queiroz93@gmail.com; Edvânia Matos de Oliveira: vaniamatoos@gmail.com

\section{Resumo}

Objetivo: Descrever o perfil epidemiológico dos casos de sífilis congênita notificados ao SUS, na cidade de Salvador/BA, entre 2007 e 2016. Métodos: Estudo descritivo, de série temporal, com dados secundários do Sistema Nacional de Agravos de Notificação (SINAN), disponibilizados pelo sistema eletrônico Tabnet Salvador. Resultados: 3.626 casos foram notificados. 99,6\% eram crianças menores de um ano; cor parda $(51,3 \%)$; escolaridade ignorada/em branco $(50,0 \%)$; residentes no distrito de São Caetano/Valéria (13,9\%). Das mulheres avaliadas, 56,6\% realizaram pré-natal, com diagnóstico neste período (40,8\%); $49,3 \%$ tiveram um tratamento inadequado e seu parceiro tratado somente em $25,5 \%$ dos casos. Das crianças avaliadas, $47,5 \%$ não realizaram exame de VDRL em líquor e nem raio-X de ossos longos (37,7\%). Conclusão: O perfil epidemiológico de sífilis congênita na cidade de Salvador no período analisado foi de crianças menores de um ano, de cor parda, que não realizaram a pesquisa do VDRL no líquor e nem radiografia de ossos longos para investigação de malformações.

Palavras-chave: sífilis congênita, transmissão vertical de doença infecciosa, doenças sexualmente transmissíveis.

\section{Abstract \\ Epidemiological profile of congenital syphilis in Salvador: 2007-2016}

Objective: To describe the epidemiological profile of cases of congenital syphilis notified to the Unified Health System, in the city of Salvador/BA, between 2007 and 2016. Methods: This is a descriptive study of temporal series, using secondary data from National Disease Notification System, provided by the electronic system Tabnet Salvador. Results: 3,626 reported cases. $99.6 \%$ were children younger than one year; brown color $(51.3 \%)$; schooling ignored/blank $(50.0 \%)$; residents in the province of São Caetano/Valeria (13.9\%). Of the women evaluated, $56.6 \%$ performed prenatal test and received diagnosis in this period (40.8\%); $49.3 \%$ had an inadequate treatment and their partner treated only in $25.5 \%$ of cases. Of the children assessed, $47.5 \%$ did not perform VDRL in cerebrospinal fluid neither X-rays of long bones $(37.7 \%)$. Conclusion: The epidemiological profile of congenital syphilis in the city of Salvador in the period analyzed was of children under one year of age, brown, who did not perform the VDRL test in CSF neither X-rays of long bones for investigation of malformations.

Key-words: congenital syphilis, vertical infectious disease transmission, sexually transmitted diseases.

\section{Resumen}

Perfil epidemiológico de la sífilis congénita en Salvador: 2007-2016

Objetivo: Describir el perfil epidemiológico de los casos de sífilis congénita notificados al Sistema Único de Salud, en la ciudad de Salvador/BA, entre 2007 y 2016. Métodos: Se realizó un estudio descriptivo de series temporales, utilizando datos secundarios de Sinan, proporcionada por el sistema electrónico Tabnet Salvador. Resultados: 3.626 casos notificados. El 99,6\% fueron niños menores de un año; de color marrón (51,3\%); escolarización 
ignorado/blanco (50,0\%), residentes en la provincia de São Caetano/Valeria (13,9\%). El 56,6\% de las mujeres evaluadas realizaron prenatal y recibieron el diagnóstico en este periodo $(40,8 \%)$; el $49,3 \%$ tuvo un tratamiento inadecuado y su pareja tratada sólo en $25,5 \%$ de los casos. El $47,5 \%$ de los niños evaluados no realizaron el VDRL en LCR y ni las radiografías de los huesos largos (37,7\%). Conclusión: El perfil epidemiológico de la ciudad de Salvador en el período analizado fue de niños menores de un año de edad, color marrón, que no realizaron el VDRL en LCR y tampoco RX de huesos largos para la investigación de malformaciones.

Palabras-clave: sífilis congénita, transmisión vertical de enfermedad infecciosa, enfermedades de transmisión sexual.

\section{Introdução}

Ainda que haja estratégias diagnósticas de baixo custo e uma terapêutica existente desde 1950, em nível global, a sífilis ainda é um preocupante problema de saúde pública, indicando uma situação epidemiológica pior que o da transmissão vertical do vírus da imunodeficiência humana (HIV) [1].

A sífilis é uma doença infecciosa, causada pelo treponema pallidum, disseminada principalmente pelo ato sexual, e que uma vez acometida à gestante, e não tratada adequadamente, ocorre a transmissão vertical. A sua ocorrência pode configurar desfechos negativos na gestação como: abortamento espontâneo, natimortos, óbito fetal, ou até sequelas que envolvem importantes órgãos e sistemas [2-5].

A transmissão da Sífilis Congênita (SC), pode processar-se em qualquer estágio da doença da mãe não tratada ou inadequadamente tratada, podendo acontecer durante o parto $\mathrm{e}$ o puerpério, na prática do aleitamento materno [3,6,7]. A maioria dos casos de SC não apresenta sintomas na fase precoce, entretanto, quando as manifestações clínicas ocorrem, estas costumam se apresentar até os dois anos de idade, sendo considerada tardia após esse período $[8,9]$.

A Organização Mundial de Saúde (OMS) afirma que mais de 1,5 milhões de mulheres grávidas são infectadas com sífilis a cada ano, e metade delas possuem filhos com algum tipo de complicação [10]. Segundo o Sistema de Agravos e Notificações (SINAN), no Brasil, foram notificados 19.228 casos de SC em menores de um ano de idade em 2015, e até junho de 2016 o Nordeste esteve em segundo lugar no ranking, como a região que possuía maior número de casos notificados de crianças com a doença [11].

$\mathrm{Na}$ Bahia, foram notificados de julho de 2007 a julho de 2016, 10.600 casos da doença em gestantes, conforme dados da Secretaria de Saúde do Estado da Bahia (SESAB) [12]. Por conseguinte, na cidade de Salvador, a ocorrência da SC apresenta crescimento anual, tendo o ano de 2016 o maior número de casos registrados [13].

Diante disso, surgiu o seguinte questionamento: Qual o perfil epidemiológico da SC no Sistema Único de Saúde (SUS), na cidade de Salvador/BA no período de 2007 a 2016?

Considerando o crescimento contínuo de casos de sífilis congênita em Salvador faz-se de fundamental importância a realização de uma pesquisa que descreva o perfil epidemiológico da doença, em virtude da necessidade de gestores e profissionais de saúde terem acesso às informações demonstrativas da significância do problema na cidade. Esses dados são fundamentais para subsidiar a elaboração de novas estratégias para detecção e tratamento precoce da doença, reduzindo consequentemente a morbimortalidade infantil.

Este estudo objetivou descrever o perfil epidemiológico dos casos de SC notificados ao SUS, na cidade de Salvador-Bahia, no período de 2007 a 2016.

Foi realizado um estudo epidemiológico, descritivo, de série temporal com dados secundários referentes aos casos de sífilis congênita notificados na cidade de Salvador/BA, no período de 2007 a 2016.

De acordo com o último Censo Demográfico disponibilizado pelo Instituto Brasileiro de Geografia e Estatística (IBGE) no ano de 2016, Salvador ocupava uma área de 692,819 km² e reunia uma população de 2.938.092 habitantes, com densidade demográfica de 3.859,44 $\mathrm{hab} / \mathrm{km}^{2}$ [14]. As informações sobre o perfil epidemiológico da sífilis congênita foram obtidas na base de dados do SINAN do SUS disponibilizados pelo Tabnet Salvador. Consideraram-se apenas os casos confirmados e notificados no referido sistema. Foram utilizadas as seguintes variáveis: faixa etária da criança, cor da pele, distrito residente, escolaridade da mãe, 
realização do pré-natal, período de diagnóstico da sífilis materna, tratamento do parceiro e realização de exames diagnósticos na criança.

A técnica de análise de dados utilizada foi a estatística descritiva. O processamento dos dados deu-se por meio do programa Excel (Microsoft@). Os resultados foram descritos por meio de valores absolutos e/ou relativos, apresentando através de gráficos e tabelas. $O$ estudo foi realizado exclusivamente com dados secundários de acesso livre, garantindo-se a preservação da identidade dos sujeitos, portanto não houve necessidade da pesquisa ser avaliada pelo comitê de ética e pesquisa.

\section{Resultados}

Em Salvador no período de 2007 a 2016, foi registrado um total de 449.628 nascidos vivos, dos quais 3.626 foram notificados com sífilis congênita, representando uma incidência da doença de 8,06 casos por mil nascidos vivos, de acordo com o gráfico 1.

Gráfico 1 - Incidência anual de sífilis congênita em Salvador/BA, 2007-2016.

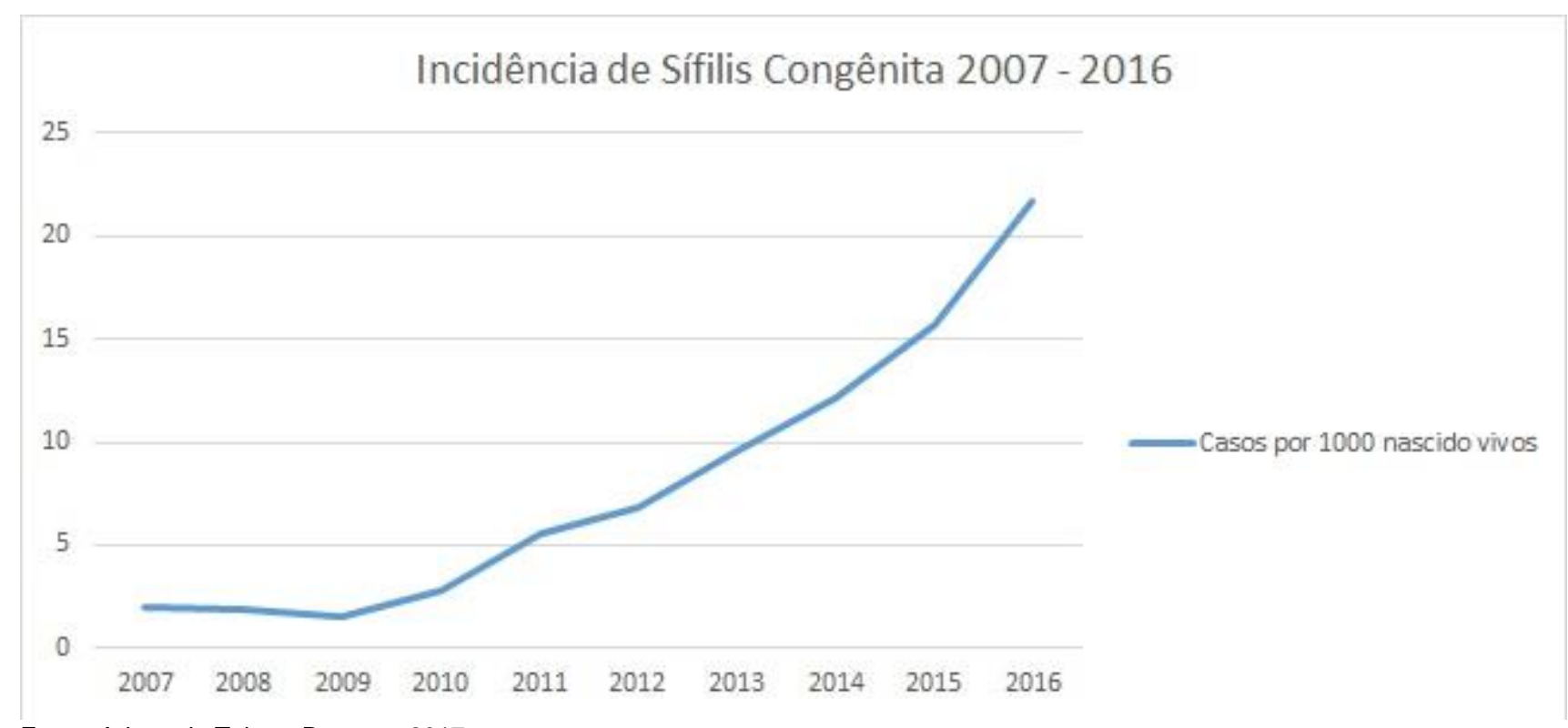

Fonte: Adaptado Tabnet-Datasus. 2017

Ao avaliar o perfil dos casos notificados de SC, a maioria era crianças menores de 1 ano e de cor parda, seguida pela preta, conforme a tabela I.

Tabela I - Distribuição das características de crianças notificadas com sífilis congênita (n=3.626), no Tabnet em Salvador/BA, 2007 a 2016.

\begin{tabular}{lll}
\hline \multicolumn{1}{c}{ Variável } & $\mathbf{N}=\mathbf{3 6 2 6}$ & $\%$ \\
\hline Faixa etária & & \\
$<1$ ano & 3614 & 99,6 \\
1 a 9 anos & 10 & 0,2 \\
$10-14$ anos & 2 & 0,2 \\
Raça & & \\
Branca & 112 & 3,0 \\
Preta & 296 & 8,1 \\
Amarela & 8 & 0,2 \\
Parda & 1861 & 51,3 \\
Indígena & 5 & 0,1 \\
Ignorada/branca & 1344 & 37,3 \\
\hline Fonte: Adaptado Tabnet - Datasus. 2017 &
\end{tabular}

Ao analisar a escolaridade materna, os registros mostraram que $50 \%$ das mulheres não tinham registro de escolaridade ou o mesmo foi ignorado. Quanto ao distrito em que residia, a 
maioria (13,8\%) referiu morar em São Caetano/Valéria, e $(12,6 \%)$ no subúrbio ferroviário, conforme a tabela II. Porém outros bairros também foram citados, como, Cabula/Beiru $(9,6 \%)$, Barra/ Pituba (9,1\%), Liberdade (6,9\%), Pau da Lima e Brotas com (6,5\%), Itapajipe $(5,7 \%)$, Itapuã (4,6\%), Centro Histórico e Cajazeiras com (3,9\%) e Boca do Rio (2,7\%).

Tabela II - Distribuição das características maternas dos casos notificados de sífilis congênita ( $n=3626)$, notificados no Tabnet em Salvador/BA, 2007 a 2016.

\begin{tabular}{lll}
\hline Variável & $\mathbf{N}=\mathbf{3 6 2 6}$ & $\%$ \\
\hline Escolaridade & & \\
Analfabeta & 12 & 0,3 \\
$1^{\circ}$ a $4^{\circ}$ série & 284 & 7,8 \\
$5^{\circ}$ a $8^{\circ}$ série & 666 & 18,3 \\
Ensino fundamental & 193 & 5,3 \\
completo & & \\
Ensino médio incompleto & 278 & 7,6 \\
Ensino médio completo & 330 & 9,1 \\
Educação superior & 23 & 0,6 \\
incompleta & & \\
Educação superior completa & 12 & 0,3 \\
lgnorado/branco & 1814 & 50,0 \\
Não se aplica & 14 & 0,7 \\
Distrito sanitário residente & & \\
São Caetano/Valéria & 501 & 13,9 \\
Subúrbio Ferroviário & 457 & 12,9 \\
lgnorado/branco & 500 & 13,8 \\
\hline
\end{tabular}

Fonte: Adaptado Tabnet - Datasus. 2017

Ao analisarmos os dados da assistência anterior ao parto, observamos que $56,6 \%$ das gestantes realizaram o pré-natal, e foram diagnosticadas durante a gestação $(40,8 \%)$, entretanto estas não realizaram o tratamento adequadamente. Dos registros sobre 0 tratamento com o parceiro, $39,6 \%$ não receberam o tratamento completo e $34,9 \%$ dos registros foram ignorados ou estavam em branco, conforme demonstrado na tabela III.

Tabela III - Distribuição das características da assistência ao pré-natal dos casos notificados de sífilis congênita ( $N=3626)$ notificados no Tabnet em Salvador/BA, 2007 a 2016.

\begin{tabular}{lll}
\hline Variável & $\mathbf{N}=\mathbf{3 6 2 6}$ & $\%$ \\
\hline Realização de pré-natal & 2053 & 56,6 \\
Sim & 620 & 17,1 \\
Não & 953 & 26,3 \\
Ignorado/branco & & \\
Período de diagnóstico da sífilis & & \\
materna & 1480 & 40,8 \\
Durante o pré-natal & 1262 & 34,8 \\
No momento do parto & 316 & 8,7 \\
Após o parto & 29 & 0,8 \\
Não realizado & 539 & 14,9 \\
Ignorado/branco & & \\
Tratamento da mãe & 725 & 19,9 \\
Adequado & 1787 & 49,3 \\
Inadequado & 492 & 13,5 \\
Não realizado & 622 & 17,3 \\
Ignorado/branco & & \\
Tratamento do parceiro & 922 & 25,5 \\
Sim & 1437 & 39,6 \\
Não & 1267 & 34,9 \\
Ignorado/branco & & \\
\hline Fonte: Adaptado Tabnet - Datasus. 2017 & &
\end{tabular}

Fonte: Adaptado Tabnet - Datasus. 2017 
Quanto ao diagnóstico da doença, os registros mostram que não foi realizado os exames de titulação Venereal Disease Research Laboratory (VDRL) em líquor nos recémnascidos $(47,5 \%)$ e nem o raio $X$ de ossos longos $(32,7 \%)$, conforme demonstrado na tabela IV.

Tabela IV - Características diagnósticas dos casos de sífilis congênita ( $n=3626)$, notificados Tabnet, em Salvador/BA, 2007 a 2016.

\begin{tabular}{lll}
\hline Variável & $\mathbf{N = 3 6 2 6}$ & $\%$ \\
\hline Alteração de VDRL em líquor & & \\
Sim & 32 & 0,5 \\
Não & 494 & 5,4 \\
Não realizado & 1569 & 47,5 \\
Ignorado/branco & 1529 & 46,6 \\
Alterações do exame de ossos & & \\
longos & & \\
Sim & 29 & 0,9 \\
Não & 851 & 24,6 \\
Não realizado & 1189 & 37,7 \\
Ignorado/branco & 1155 & 36,8 \\
\hline Fo:Adaro
\end{tabular}

Fonte: Adaptado Tabnet - Datasus. 2017

Discussão

As ocorrências de SC na cidade merecem uma atenção específica, uma vez que a incidência da doença é crescente no período analisado. Somente em 2016, foram registrados 21,75 casos para cada mil nascidos vivos, o que representa um valor extremamente acima dos parâmetros nacionais [11]. Este elevado crescimento anual das notificações deu-se provavelmente, em virtude da implantação da Portaria SS no 41 de 24/03/2005, que teve como objetivo realizar o teste não treponêmico VDRL no primeiro atendimento, na $30^{\mathrm{a}}$ semana de gestação, como também sua repetição para um controle da terapêutica no momento da internação para o parto ou curetagem, conforme recomendado pelo Ministério da Saúde (MS).

Na presente pesquisa, o percentual de crianças menores de um ano com a doença é o mais elevado. Estudos $[3,15]$ mostram que essa incidência provavelmente está relacionada à apresentação precoce de sinais da doença, sendo identificados facilmente nessa fase. Este achado ganha representatividade ao analisarmos os boletins epidemiológicos [11], mostrando a elevada incidência da doença nessa idade, em diversas regiões do Brasil.

Ao analisarmos a etnia, observamos a predominância dos registros de crianças de raça/cor parda e preta, achado que corrobora outros autores [16-19]. O estudo de Lima et al. [20] mostrou a predominância desse achado na cidade de Belo Horizonte, destacando que as mulheres negras ainda possuem dificuldades socioeconômicas que contribuíram para a transmissão vertical. O que supostamente aponta que as desigualdades sociais estão incluídas em distintos riscos para a SC.

Essa vulnerabilidade social também está representada pelo baixo nível de escolaridade dessas mulheres, achado que está presente em outros estudos [16,18]. Entretanto, grande parte dos casos notificados da doença possui o registro de escolaridade materna ignorada ou em branco, aspecto que possivelmente reflete o descuido do profissional na realização do registro. Os dados relativos ao perfil de escolaridade possibilitam a compreensão sobre quais aspectos e contextos essas mulheres estão inseridas, para que assim sejam identificadas as falhas assistenciais e o planejamento de ações necessárias.

Referente ao distrito sanitário que a genitora reside, observamos que assim como outros estudos $[19,20]$, as mulheres que residem em periferias e subúrbios possuem uma condição que provavelmente aumenta o risco de transmissão vertical. De acordo com Melo et al. [16], este risco ocorre em virtude dessas gestantes buscarem a atenção pré-natal tardiamente, ou, é presumível que este achado pode estar relacionado aos reduzidos números de profissionais e unidades básicas de saúde nestes distritos, a exemplo de São Caetano/Valéria que, segundo a Secretaria Municipal da Saúde de Salvador (SMS), possui apenas sete Unidades de Saúde da Família (USF) e três Unidades Básicas de Saúde (UBS), comportando mais de 249.238 habitantes. Sendo assim, é possível que esses aspectos interfiram na qualidade da assistência ao binômio mãe-feto [21].

Apesar de os registros demonstrarem que a maioria das mães possuía 0 acompanhamento durante o pré-natal, as taxas de neonatos infectados com a doença são 
elevadas ao longo dos anos. Esses resultados podem estar relacionados à reestruturação da atenção pré-natal, com o treinamento dos profissionais de saúde, para que resulte na identificação da doença precocemente.

Neste estudo, a maioria das mulheres foi diagnosticada ainda na gestação, entretanto 1.262 casos foram diagnosticados durante o parto, aspecto que pode relacionar-se à infecção próxima ao parto ou ainda pela terapêutica inadequada. Possivelmente, esses registros de casos em 2016 [11,22], estejam relacionados à falha na terapêutica, em virtude da escassez de Penicilina Benzatina. A ausência de matéria prima inviabilizou a formulação da droga e consequente distribuição, aspectos que impediram o tratamento adequado dessas mulheres.

Ao analisarmos a realização do tratamento pelo parceiro, observamos que a maioria deles não o realizou, achado também encontrado em outros estudos [10,23]. Acreditamos que este achado seja em virtude da não inserção do homem no acompanhamento pré-natal. Apesar do profissional de saúde explicar para a gestante a importância e a necessidade do tratamento do parceiro para evitar sua reinfecção não se sabe se o parceiro adota as condutas orientadas e o tratamento concomitante com a gestante. $O$ tratamento é fundamental durante a gestação, entretanto, é primordial que o parceiro também realize a terapêutica [22,24]. Dessa forma, faz-se necessário a inserção do companheiro no pré-natal, como estratégia de melhoria na adesão ao tratamento.

Foi demonstrado nesta pesquisa, que o exame de coleta de líquor e o raio $\mathrm{X}$ de ossos longos, não foram procedimentos realizados nos neonatos, sendo estes dados também encontrados em outros estudos [18,25]. Cavalcante et al. [1] relataram que o exame de líquor é essencial para a investigação da neurosífilis e $o$ raio $X$ de ossos longos para diagnosticar deformações recorrentes da doença, como, osteomielite, periostite e osteocondrite. Portanto, a não realização destes implica na prorrogação de investigações e condutas precoces acerca das alterações encontradas no exame.

Conclusão

A pesquisa demonstrou que o perfil epidemiológico de SC na cidade de Salvador no período analisado foi de: crianças menores de um ano, na cor parda, que não realizaram a pesquisa do VDRL no líquor e nem RX de ossos longos para investigação de malformações. Estes eram filhos de pais que não realizaram o tratamento; de mães com escolaridade entre $5^{a}$ e $8^{a}$ série, residentes do distrito sanitário de São Caetano/Valéria, que realizaram o pré-natal, tiveram o diagnóstico da infecção nesta assistência, entretanto não realizaram o tratamento adequado.

A limitação deste estudo deu-se em virtude das variáveis apresentarem classificações como "ignorado/em branco", o que representa prováveis sub-registros e subnotificações dos casos. Destaca-se dessa forma a necessidade da preparação dos profissionais de saúde responsáveis pela manipulação dessas fichas para obtenção de dados mais fidedignos. Diante disso, é evidenciada a relevância da vigilância epidemiológica da SC, pois o acompanhamento das notificações é indispensável para que as próximas pesquisas sobre o perfil epidemiológico obtenham dados exatos e completos e ofereçam informações fundamentais para 0 planejamento das intervenções no controle da doença nos municípios, a fim de reduzir o número de casos.

\section{Referências}

1. Cavalcante PAM, Pereira RBL, Castro JGD. Sífilis gestacional e congênita em Palmas, Tocantins, 2007-2014. Epidemiol Serv Saúde 2017;26(2):255-64.

2. Hook EW. Syphilis. The Lancet 2016;389(10078):1550-7.

3. Informes Técnicos Institucionais. Sífilis congênita e sífilis na gestação. Rev Saúde Pública 2008;42(4):768-72.

4. Lima VC, Mororó M, Feijão DM, Frota MVV, Martins MA, Ribeiro SM, et al. Percepção de mães acerca da sífilis congênita em seu concepto. Rev de Saúde Pública do Paraná 2016;17(2):118-25.

5. Santos GC, Paluch LRB, Cerqueira TPS, Passos NCR. Prevalência e fatores associados à sífilis em gestantes atendidas pelo SUS em município da Bahia. Rev Baiana de Saúde Pública 2015;39(3):529-41.

6. Carvalho IS, Brito RS. Sífilis congênita no Rio Grande do Norte: estudo descritivo do período 2007-2010. Epidemiol Serv Saúde 2014;23(2):287-94. 
7. Araújo EC, Costa KSG, Silva RS, Azevedo VNG, Lima AS. Importância do pré-natal na prevenção da Sífilis Congênita. Rev Para Med 2006;20(1):47-51.

8. Avelleira JCR, Bottino G. Sífilis: diagnóstico, tratamento e controle. An Bras Dermatol 2006;81(2):111-26.

9. Magalhães DMS, Kawaguchi IAL, Dias A, Calderon IMP.sífilis na gestação e sua influência na morbimortalidade materno-infantil. Com Ciências Saúde 2011;22(1):43-54.

10. Cooper JM, Michelow IC, Wozniak PS, Sanchez PJ. Em tempo: a persistência da sífilis congênita no Brasil - Mais avanços são necessários. Rev Paul Pediatr 2016;34(3):2513.

11. Ministério da saúde (BR). Secretaria de Vigilância em Saúde. Boletim Epidemiológico 2016;47(35):28.

12. Bahia, Secretaria de estado da saúde. Dia Nacional de Combate à Sífilis terá atividade na próxima sexta-feira. Salvador; 2016 [citado 2017 jun. 8]. Disponível em:

http://www.saude.ba.gov.br/novoportal/index.php?option=com_content\&view=article\&id $=11162$ :-dia-nacional-de-combate-a-sifilis-tera-atividade-na-proxima-sextafeira\&catid=13: noticias\&ltemid $=25$.

13. Salvador, Secretaria Municipal de Saúde. TABNET Salvador. Salvador; 2017 [citado 2017 Abr 12]. Disponível em: http://www.tabnet.saude.salvador.ba.gov.br/.

14. Brasil. Instituto Brasileiro de Geografia e Estatística. Estatísticas populacionais. 2016 [citado 2017 Maio 12]. Disponível em: http://cidades.ibge.gov.br/xtras/perfil.php?codmun=292740.

15. Brasil, Ministério da Saúde, Secretaria de Vigilância em Saúde, Programa Nacional de DST e Aids. Diretrizes para o Controle da Sífilis Congênita, Manual de Bolso. Brasília; 2006 [citado 2017 Mar 12]. Disponível em: http://bvsms.saude.gov.br/bvs/publicacoes/manual_sifilis_bolso.pdf.

16. Melo NGDO, Filho DAM, Ferreira LOC. Diferenciais intraurbanos de sífilis congênita no Recife, Pernambuco, Brasil (2004-2006). Epidemiol Serv Saúde 2011;20(2):213-22.

17. Leal MC, Gama SGN, Cunha CB. Desigualdades raciais, sociodemográficas e na assistência ao pré-natal e ao parto, 1999-2001. Rev Saúde Pública 2005;39(1):100-7.

18. Holanda MTCG, Barreto MA, Machado KMM, Pereira RC. Perfil epidemiológico da sífilis congênita no Município do Natal, Rio Grande do Norte - 2004 a 2007. Epidemiol Serv Saúde 2011;20(2):203-12.

19. Nonato SM, Melo APS, Guimarães MDC. Sífilis na gestação e fatores associados à sífilis congênita em Belo Horizonte-MG, 2010-2013. Epidemiol Serv Saúde 2015;24(4):681-94.

20. Lima MG, Santos RFR, Barbosa GJA, Ribeiro GS. Incidência e fatores de risco para sífilis congênita em Belo Horizonte, Minas Gerais, 2001-2008. Ciênc Saúde Coletiva 2013;18(2):499-506.

21. Salvador, Prefeitura Municipal. SMS - Secretaria Municipal da Saúde de Salvador. Salvador; 2016. Disponível em: http://www.saude.salvador.ba.gov.br/distritossanitarios/\#1463074808908-687063f8-c12e.

22. Bahia, Superintendência de vigilância e proteção da saúde de Salvador. Informe Epidemiológico da Sífilis. Salvador; 2016 [citado 2017 Maio 12]. Disponível em: http://www.suvisa.ba.gov.br/sites/default/files/Informe\%20Epidemiol\%C3\%B3gico\%20S \%C3\%ADfilis\%20Final\%202016.pdf.

23. Holanda MTCG, Barreto MA, Machado KMM, Pereira RC. Perfil epidemiológico da sífilis congênita no Município do Natal, Rio Grande do Norte 2004 a 2007. Epidemiol Serv Saúde 2011;20(2):203-12.

24. Milanez H, Amaral E. Por que ainda não conseguimos controlar o problema da sífilis em gestantes e recém-nascidos? Rev Bras Ginecol Obstet 2008;30(7):325-7.

25. Almeida MFG, Pereira SM. Caracterização Epidemiológica da Sífilis Congênita no município de Salvador, DST. J Bras Doenças Sex Transm 2007;19(3-4):144-56. 